\title{
Correction to: Geochemistry of ultramafic, mafic, and felsic xenoliths from the Gölcük (Isparta, SW Turkey) alkali rocks: genetic relationship with arc magmas
}

Kamil Yılmaz ${ }^{1}$

Published online: 28 May 2019

(C) Saudi Society for Geosciences 2019

Correction to: Arabian Journal of Geosciences (2019) 12: 306 https://doi.org/10.1007/s12517-019-4461-6

The original version of this paper was published with error. Table 1 consists of five sheets but only one was processed, therefore four sheets were missing. Given in this article is the corrected table.

The online version of the original article can be found at https://oi.org/ $10.1007 / \mathrm{s} 12517-019-4461-6$

Kamil Y1lmaz

kamilyilmaz@sdu.edu.tr

Faculty of Engineering, Department of Geological Engineering,

Süleyman Demirel University, Isparta, Turkey 
Table 1 Whole-rock major (wt \%) and trace (ppm) element analyses of representative samples of the host rocks, ultramafic, mafic, and felsic xenoliths from the Gölcük volcanics. $\mathrm{Mg \#}=$ atomic $100 \times(\mathrm{Mg} / \mathrm{Mg}+$
$\mathrm{Fe}^{2+}$ ), assuming $\mathrm{Fe}_{2} \mathrm{O}_{3} / \mathrm{FeO}=0.1$. Chemical compositions of host rocks are from Elitok et al. (2010)

\begin{tabular}{|c|c|c|c|c|c|c|c|c|c|c|}
\hline Sample & KY-01 & KY-03 & KY-05 & KY-19 & KY-20 & KY-22 & KY-12 & KY-16 & KY-17 & KY-21 \\
\hline Xenolith & Ultramafic & & & & & & Mafic & & & \\
\hline $\mathrm{SiO}_{2}(\mathrm{wt} . \%)$ & 43.92 & 44.53 & 37.46 & 41.59 & 43.41 & 39.13 & 46.88 & 47.79 & 48.82 & 45.93 \\
\hline $\mathrm{Al}_{2} \mathrm{O}_{3}$ & 11.69 & 12.94 & 12.86 & 7.91 & 9.06 & 8.00 & 8.01 & 6.15 & 12.64 & 13.49 \\
\hline $\mathrm{Fe}_{2} \mathrm{O}_{3}$ & 7.61 & 10.03 & 4.95 & 10.31 & 11.30 & 7.88 & 8.82 & 5.74 & 7.62 & 9.27 \\
\hline $\mathrm{MgO}$ & 10.06 & 8.37 & 25.93 & 13.99 & 13.71 & 14.64 & 10.61 & 16.96 & 8.45 & 7.87 \\
\hline $\mathrm{CaO}$ & 15.03 & 14.52 & 1.21 & 14.23 & 11.09 & 16.63 & 17.33 & 17.44 & 11.88 & 12.46 \\
\hline $\mathrm{Na}_{2} \mathrm{O}$ & 1.86 & 2.46 & 0.63 & 0.65 & 0.61 & 0.52 & 1.84 & 0.56 & 2.88 & 2.44 \\
\hline $\mathrm{K}_{2} \mathrm{O}$ & 3.45 & 2.14 & 7.44 & 4.29 & 4.90 & 4.29 & 2.05 & 2.47 & 3.26 & 2.67 \\
\hline $\mathrm{TiO}_{2}$ & 1.19 & 1.29 & 0.36 & 2.09 & 1.84 & 1.47 & 0.76 & 0.85 & 0.90 & 1.21 \\
\hline $\mathrm{P}_{2} \mathrm{O}_{5}$ & 3.23 & 2.04 & 1.26 & 2.84 & 1.35 & 5.40 & 2.01 & 0.22 & 1.61 & 1.35 \\
\hline $\mathrm{MnO}$ & 0.11 & 0.17 & 0.43 & 0.19 & 0.18 & 0.10 & 0.22 & 0.08 & 0.14 & 0.22 \\
\hline LOI & 0.8 & 0.4 & 1.5 & 0.8 & 1.2 & 0.8 & 0.7 & 0.8 & 0.5 & 1.9 \\
\hline Total & 98.95 & 98.93 & 94.04 & 98.97 & 98.78 & 98.92 & 99.28 & 99.27 & 98.74 & 98.88 \\
\hline $\mathrm{Mg \#}$ & 73.0 & 63.0 & 91.0 & 73.0 & 71.0 & 79.0 & 71.0 & 86.0 & 69.0 & 63.0 \\
\hline $\mathrm{Sr}(\mathrm{ppm})$ & 2637.0 & 4079.0 & 618.0 & 1061.0 & 660.0 & 1634.0 & 1943.0 & 872.8 & 5165.0 & 3954.0 \\
\hline $\mathrm{Ba}$ & 3465.0 & 1525.0 & 13922.0 & 3431.0 & 6740.0 & 3689.0 & 934.0 & 2525.0 & 2586.0 & 2693.0 \\
\hline $\mathrm{Rb}$ & 82.3 & 60.6 & 578.5 & 139.2 & 181.9 & 149.6 & 91.4 & 65.2 & 117.6 & 95.9 \\
\hline Cs & 1.8 & 1.4 & 16.6 & 6.5 & 11.0 & 13.6 & 1.7 & 1.2 & 2.2 & 36.5 \\
\hline $\mathrm{Ga}$ & 13.1 & 19.3 & 13.7 & 13.0 & 15.9 & 10.0 & 14.7 & 6.9 & 16.2 & 17.2 \\
\hline $\mathrm{Hf}$ & 5.6 & 10.1 & 7.0 & 16.9 & 1.6 & 2.2 & 4.5 & 1.1 & 5.6 & 6.6 \\
\hline $\mathrm{Ni}$ & 26.6 & 18.4 & 13.9 & 133.1 & 157.9 & 74.3 & 28.6 & 68.3 & 45.5 & 16.6 \\
\hline $\mathrm{Sc}$ & 37 & 29 & 4 & 36 & 32 & 27 & 46 & 55 & 25 & 32 \\
\hline $\mathrm{Cr}$ & 48 & 48 & 55 & 582 & 582 & 89 & 144 & 1383 & 157 & 82 \\
\hline $\mathrm{Zr}$ & 177.9 & 322.6 & 277.5 & 789.3 & 51.3 & 65.4 & 134.8 & 44.3 & 213.2 & 261.9 \\
\hline $\mathrm{Y}$ & 26.4 & 42.8 & 402.1 & 39.9 & 25.7 & 40.1 & 31.2 & 7.8 & 33.1 & 34.9 \\
\hline $\mathrm{Nb}$ & 23.8 & 28.9 & 50.8 & 39.9 & 34.3 & 16.8 & 11.0 & 4.1 & 24.7 & 45.3 \\
\hline $\mathrm{Ta}$ & 1.0 & 1.4 & 1.0 & 1.4 & 0.8 & 0.5 & 0.2 & 0.3 & 1.1 & 1.4 \\
\hline Th & 34.2 & 33.0 & 132.3 & 13.6 & 5.5 & 18.9 & 16.5 & 3.3 & 26.3 & 22.4 \\
\hline $\mathrm{U}$ & 6.7 & 6.0 & 248.7 & 2.6 & 2.1 & 4.3 & 4.2 & 0.5 & 6.0 & 4.3 \\
\hline $\mathrm{Pb}$ & 3.5 & 3.5 & 9.3 & 1.7 & 2.0 & 2.0 & 1.9 & 3.7 & 2.1 & 6.6 \\
\hline $\mathrm{La}$ & 193.3 & 281.5 & 11036 & 232.5 & 130.1 & 321.3 & 232.5 & 30.7 & 249.7 & 198.6 \\
\hline $\mathrm{Ce}$ & 374.3 & 584.7 & 16201 & 438.3 & 275.9 & 626.2 & 443.5 & 63.9 & 501.5 & 402.9 \\
\hline $\operatorname{Pr}$ & 43.38 & 70.39 & 1179 & 49.03 & 32.19 & 71.61 & 50.29 & 8.83 & 57.38 & 46.96 \\
\hline $\mathrm{Nd}$ & 160.7 & 276.8 & 2986 & 180.8 & 127.4 & 289.4 & 187.2 & 39.7 & 221.0 & 181.1 \\
\hline $\mathrm{Sm}$ & 25.21 & 40.25 & 245.5 & 26.67 & 18.48 & 37.88 & 26.04 & 6.76 & 32.03 & 26.17 \\
\hline $\mathrm{Eu}$ & 5.91 & 9.33 & 63.28 & 6.60 & 4.07 & 9.22 & 6.03 & 1.89 & 7.64 & 5.93 \\
\hline $\mathrm{Gd}$ & 16.60 & 22.77 & 156.3 & 19.16 & 12.40 & 24.13 & 15.92 & 4.86 & 19.97 & 16.30 \\
\hline $\mathrm{Tb}$ & 1.63 & 2.60 & 13.78 & 2.30 & 1.36 & 2.32 & 1.67 & 0.51 & 2.00 & 1.85 \\
\hline Dy & 6.31 & 10.91 & 58.85 & 10.14 & 5.69 & 9.68 & 6.81 & 2.28 & 7.50 & 8.86 \\
\hline Ho & 0.86 & 1.43 & 9.22 & 1.61 & 0.99 & 1.21 & 1.15 & 0.29 & 1.18 & 1.24 \\
\hline $\mathrm{Er}$ & 2.63 & 3.73 & 29.21 & 3.88 & 2.25 & 3.16 & 2.33 & 0.67 & 2.85 & 3.54 \\
\hline $\mathrm{Tm}$ & 0.29 & 0.52 & 4.26 & 0.47 & 0.30 & 0.41 & 0.37 & 0.08 & 0.37 & 0.46 \\
\hline $\mathrm{Yb}$ & 1.61 & 2.91 & 26.79 & 3.10 & 2.01 & 2.23 & 2.16 & 0.43 & 2.40 & 3.01 \\
\hline $\mathrm{Lu}$ & 0.21 & 0.41 & 3.31 & 0.44 & 0.26 & 0.28 & 0.34 & 0.06 & 0.34 & 0.43 \\
\hline$(\mathrm{La} / \mathrm{Sm})_{\mathrm{N}}$ & 4.83 & 4.40 & 28.3 & 5.49 & 4.43 & 5.34 & 5.62 & 2.86 & 4.91 & 4.78 \\
\hline$(\mathrm{Gd} / \mathrm{Yb})_{\mathrm{N}}$ & 8.36 & 6.34 & 4.73 & 5.01 & 5.00 & 8.77 & 5.97 & 9.16 & 6.74 & 4.39 \\
\hline
\end{tabular}


Table 1 (continued)

\begin{tabular}{|c|c|c|c|c|c|c|c|c|c|c|c|}
\hline$\overline{\mathrm{Eu} / \mathrm{Eu}^{*}}$ & 0.88 & 0.94 & 0.99 & 0.89 & 0.82 & 0.93 & 0.91 & 1.01 & 0.92 & 0.88 & \\
\hline $\mathrm{La}_{\mathrm{N}}$ & 527 & 767 & 30071 & 634 & 354 & 875 & 634 & 84 & 680 & 541 & \\
\hline $\mathrm{Yb}_{\mathrm{N}}$ & 6.5 & 11.7 & 108.0 & 12.5 & 8.1 & 9.0 & 8.7 & 1.7 & 9.7 & 12.1 & \\
\hline$(\mathrm{La} / \mathrm{Yb})_{\mathrm{N}}$ & 81.1 & 65.4 & 278.4 & 50.7 & 43.7 & 97.4 & 72.7 & 48.2 & 70.3 & 44.6 & \\
\hline $\mathrm{Ba} / \mathrm{Nb}$ & 145.58 & 52.76 & 274.05 & 86.00 & 196.50 & 219.58 & 84.90 & 615.85 & 104.69 & 59.45 & \\
\hline $\mathrm{La} / \mathrm{Nb}$ & 8.12 & 9.74 & 217.24 & 5.83 & 3.79 & 19.12 & 21.13 & 7.49 & 10.11 & 4.38 & \\
\hline $\mathrm{Rb} / \mathrm{Nb}$ & 3.5 & 2.1 & 11.4 & 3.5 & 5.3 & 8.9 & 8.3 & 15.9 & 4.8 & 2.1 & \\
\hline $\mathrm{Th} / \mathrm{Ta}$ & 34.20 & 23.57 & 132.30 & 9.71 & 6.87 & 37.80 & 82.50 & 11.00 & 23.91 & 16.00 & \\
\hline $\mathrm{Th} / \mathrm{Nb}$ & 1.43 & 1.14 & 2.60 & 0.34 & 0.16 & 1.13 & 1.50 & 0.80 & 1.06 & 0.49 & \\
\hline $\mathrm{Rb} / \mathrm{Sr}$ & 0.031 & 0.015 & 0.936 & 0.131 & 0.276 & 0.092 & 0.047 & 0.075 & 0.023 & 0.024 & \\
\hline Sample & KY-23 & KY-25 & KY-04 & KY-06 & KY-07 & KY-08 & KY-09 & KY-13 & KY-14 & KY-15 & KY-18 \\
\hline Xenolith & Mafic & & Felsic & & & & & & & & \\
\hline $\mathrm{SiO}_{2}$ (wt.\%) & 47.30 & 49.68 & 58.76 & 54.59 & 61.92 & 59.42 & 60.09 & 53.32 & 57.34 & 50.80 & 59.48 \\
\hline $\mathrm{Al}_{2} \mathrm{O}_{3}$ & 7.06 & 5.31 & 16.11 & 16.83 & 17.67 & 17.52 & 18.35 & 17.99 & 17.82 & 17.67 & 17.25 \\
\hline $\mathrm{Fe}_{2} \mathrm{O}_{3}$ & 6.47 & 6.63 & 3.02 & 6.02 & 3.22 & 4.71 & 3.81 & 6.32 & 5.23 & 6.04 & 3.55 \\
\hline $\mathrm{MgO}$ & 16.46 & 14.45 & 3.08 & 3.15 & 1.34 & 1.49 & 1.56 & 3.06 & 1.73 & 1.65 & 1.79 \\
\hline $\mathrm{CaO}$ & 14.51 & 18.21 & 8.10 & 7.14 & 2.13 & 3.75 & 3.71 & 6.88 & 4.66 & 5.49 & 3.98 \\
\hline $\mathrm{Na}_{2} \mathrm{O}$ & 0.57 & 0.93 & 4.95 & 4.78 & 5.70 & 5.34 & 6.28 & 4.01 & 4.53 & 5.27 & 4.45 \\
\hline $\mathrm{K}_{2} \mathrm{O}$ & 3.70 & 1.77 & 3.41 & 3.77 & 6.05 & 5.09 & 3.99 & 4.95 & 5.50 & 3.93 & 5.94 \\
\hline $\mathrm{TiO}_{2}$ & 1.10 & 0.66 & 0.38 & 0.79 & 0.54 & 0.64 & 0.49 & 0.87 & 0.67 & 0.81 & 0.44 \\
\hline $\mathrm{P}_{2} \mathrm{O}_{5}$ & 0.63 & 0.46 & 0.30 & 0.72 & 0.21 & 0.27 & 0.29 & 0.74 & 0.31 & 0.31 & 0.33 \\
\hline $\mathrm{MnO}$ & 0.09 & 0.16 & 0.17 & 0.13 & 0.07 & 0.10 & 0.08 & 0.12 & 0.11 & 0.12 & 0.09 \\
\hline LOI & 1.2 & 0.9 & 0.4 & 1.0 & 0.3 & 0.5 & 0.5 & 0.4 & 0.6 & 0.4 & 1.8 \\
\hline Total & 99.21 & 99.32 & 98.69 & 98.90 & 99.14 & 98.83 & 99.13 & 98.64 & 98.47 & 98.47 & 99.07 \\
\hline Mg\# & 84.0 & 81.0 & 67.0 & 51.0 & 46.0 & 39.0 & 45.0 & 49.0 & 40.0 & 35.0 & 50.0 \\
\hline $\mathrm{Sr}(\mathrm{ppm})$ & 905.1 & 1242.0 & 5724.0 & 4750.0 & 3041.0 & 5040.0 & 3738.0 & 5308.0 & 6464.0 & 6967.0 & 3448.0 \\
\hline $\mathrm{Ba}$ & 2590.0 & 1576.0 & 2852.0 & 2304.0 & 2291.0 & 2888.0 & 2111.0 & 3929.0 & 4281.0 & 4016.0 & 2696.0 \\
\hline $\mathrm{Rb}$ & 104.9 & 55.5 & 50.9 & 85.2 & 120.0 & 69.7 & 72.6 & 80.0 & 74.0 & 49.5 & 149.9 \\
\hline $\mathrm{Cs}$ & 9.9 & 4.0 & 0.3 & 3.1 & 0.9 & 0.4 & 0.9 & 0.5 & 0.2 & 0.2 & 3.6 \\
\hline $\mathrm{Ga}$ & 8.5 & 8.4 & 21.1 & 22.0 & 23.6 & 19.6 & 22.5 & 23.4 & 21.1 & 21.3 & 20.7 \\
\hline Hf & 2.5 & 3.3 & 8.7 & 9.1 & 13.3 & 10.1 & 9.0 & 7.3 & 16.6 & 5.4 & 10.5 \\
\hline $\mathrm{Ni}$ & 132.8 & 59.7 & 0.9 & 4.4 & 5.9 & 4.5 & 4.7 & 2.2 & 1.6 & 1.6 & 6.4 \\
\hline $\mathrm{Sc}$ & 33 & 40 & 4 & 13 & 3 & 4 & 6 & 10 & 5 & 5 & 6 \\
\hline $\mathrm{Cr}$ & 541 & 958 & 21 & 34 & 21 & 14 & 14 & 14 & 14 & 14 & 41 \\
\hline $\mathrm{Zr}$ & 80.9 & 83.7 & 353.5 & 366.3 & 600.1 & 453.1 & 421.3 & 353.4 & 854.0 & 185.3 & 397.2 \\
\hline $\mathrm{Y}$ & 14.7 & 17.8 & 34.3 & 26.5 & 27.0 & 28.1 & 18.2 & 26.3 & 31.5 & 32.8 & 21.8 \\
\hline $\mathrm{Nb}$ & 17.9 & 10.8 & 55.7 & 48.7 & 52.7 & 63.5 & 40.8 & 58.1 & 61.0 & 72.7 & 68.3 \\
\hline $\mathrm{Ta}$ & 0.5 & 0.7 & 2.4 & 1.6 & 2.7 & 3.5 & 2.0 & 2.3 & 3.7 & 4.4 & 3.0 \\
\hline Th & 8.0 & 5.4 & 52.3 & 65.3 & 47.8 & 25.5 & 52.7 & 58.4 & 28.9 & 48.6 & 71.2 \\
\hline $\mathrm{U}$ & 1.3 & 1.0 & 8.7 & 17.4 & 9.6 & 6.7 & 11.2 & 15.0 & 8.0 & 7.9 & 20.4 \\
\hline $\mathrm{Pb}$ & 1.0 & 0.9 & 4.5 & 5.7 & 2.0 & 1.9 & 0.9 & 6.0 & 1.6 & 1.5 & 8.1 \\
\hline $\mathrm{La}$ & 83.4 & 62.1 & 427.5 & 190.1 & 183.2 & 175.3 & 111.5 & 222.6 & 127.1 & 236.6 & 179.3 \\
\hline $\mathrm{Ce}$ & 187.4 & 134.6 & 629.6 & 351.0 & 309.2 & 366.3 & 218.0 & 397.6 & 292.2 & 438.6 & 300.1 \\
\hline $\operatorname{Pr}$ & 23.32 & 17.37 & 57.62 & 38.80 & 31.53 & 41.43 & 23.19 & 43.11 & 36.68 & 46.74 & 30.01 \\
\hline $\mathrm{Nd}$ & 94.3 & 75.1 & 180.8 & 138.1 & 104.6 & 146.5 & 79.9 & 153.6 & 135.4 & 157.6 & 98.5 \\
\hline $\mathrm{Sm}$ & 13.89 & 11.76 & 22.82 & 19.79 & 14.32 & 20.81 & 11.68 & 21.31 & 20.02 & 22.14 & 12.41 \\
\hline $\mathrm{Eu}$ & 3.66 & 2.74 & 4.85 & 4.75 & 3.28 & 5.16 & 2.89 & 5.30 & 5.34 & 5.40 & 3.21 \\
\hline $\mathrm{Gd}$ & 8.72 & 7.34 & 12.95 & 12.35 & 8.99 & 12.29 & 7.87 & 12.57 & 12.64 & 13.67 & 7.53 \\
\hline $\mathrm{Tb}$ & 0.95 & 0.83 & 1.42 & 1.34 & 1.10 & 1.42 & 0.89 & 1.41 & 1.50 & 1.55 & 0.95 \\
\hline Dy & 3.70 & 4.16 & 6.44 & 5.79 & 4.77 & 6.65 & 3.64 & 5.26 & 6.48 & 6.50 & 4.24 \\
\hline Ho & 0.54 & 0.57 & 1.05 & 0.96 & 0.93 & 0.91 & 0.75 & 0.76 & 1.14 & 1.19 & 0.70 \\
\hline $\mathrm{Er}$ & 1.29 & 1.62 & 3.07 & 2.54 & 2.83 & 2.70 & 2.16 & 2.67 & 3.05 & 2.69 & 2.23 \\
\hline $\mathrm{Tm}$ & 0.17 & 0.22 & 0.51 & 0.35 & 0.40 & 0.40 & 0.29 & 0.31 & 0.39 & 0.42 & 0.33 \\
\hline $\mathrm{Yb}$ & 1.04 & 1.47 & 3.19 & 2.30 & 2.38 & 2.25 & 1.73 & 1.86 & 2.55 & 2.54 & 2.02 \\
\hline $\mathrm{Lu}$ & 0.14 & 0.19 & 0.46 & 0.34 & 0.42 & 0.40 & 0.24 & 0.31 & 0.36 & 0.34 & 0.32 \\
\hline$(\mathrm{La} / \mathrm{Sm})_{\mathrm{N}}$ & 3.78 & 3.32 & 11.79 & 6.05 & 8.05 & 5.30 & 6.01 & 6.57 & 4.00 & 6.73 & 9.09 \\
\hline$(\mathrm{Gd} / \mathrm{Yb})_{\mathrm{N}}$ & 6.80 & 4.05 & 3.29 & 4.35 & 3.06 & 4.43 & 3.69 & 5.48 & 4.02 & 4.36 & 3.02 \\
\hline $\mathrm{Eu} / \mathrm{Eu}^{*}$ & 1.02 & 0.90 & 0.86 & 0.93 & 0.88 & 0.99 & 0.92 & 1.00 & 1.03 & 0.95 & 1.01 \\
\hline $\mathrm{La}_{\mathrm{N}}$ & 227 & 169 & 1165 & 518 & 499 & 478 & 304 & 607 & 346 & 645 & 489 \\
\hline $\mathrm{Yb}_{\mathrm{N}}$ & 4.2 & 5.9 & 12.9 & 9.3 & 9.6 & 9.1 & 7.0 & 7.5 & 10.3 & 10.2 & 8.1 \\
\hline$(\mathrm{La} / \mathrm{Yb})_{\mathrm{N}}$ & 54.2 & 28.5 & 90.6 & 55.9 & 52.0 & 52.6 & 43.6 & 80.9 & 33.7 & 62.9 & 60.0 \\
\hline $\mathrm{Ba} / \mathrm{Nb}$ & 144.69 & 145.92 & 51.20 & 47.31 & 43.47 & 45.48 & 51.74 & 67.62 & 70.18 & 55.24 & 39.47 \\
\hline $\mathrm{La} / \mathrm{Nb}$ & 4.66 & 5.75 & 7.67 & 3.90 & 3.47 & 2.76 & 2.73 & 3.83 & 2.08 & 3.25 & 2.62 \\
\hline
\end{tabular}


Table 1 (continued)

\begin{tabular}{|c|c|c|c|c|c|c|c|c|c|c|c|}
\hline$\overline{\mathrm{Rb} / \mathrm{Nb}}$ & 5.9 & 5.1 & 0.9 & 1.7 & 2.3 & 1.1 & 1.8 & 1.4 & 1.2 & 0.7 & 2.2 \\
\hline $\mathrm{Th} / \mathrm{Ta}$ & 16.00 & 7.71 & 21.79 & 40.81 & 17.70 & 7.28 & 26.35 & 25.39 & 7.81 & 11.04 & 23.73 \\
\hline $\mathrm{Th} / \mathrm{Nb}$ & 0.45 & 0.50 & 0.94 & 1.34 & 0.91 & 0.40 & 1.29 & 1.00 & 0.47 & 0.67 & 1.04 \\
\hline $\mathrm{Rb} / \mathrm{Sr}$ & 0.016 & 0.045 & 0.009 & 0.018 & 0.039 & 0.014 & 0.019 & 0.015 & 0.011 & 0.007 & 0.043 \\
\hline Sample & G1-8 & Gl-29 & Gl-39 & Gl-45 & G1-47 & G1-93 & GP-11 & GP-12 & & & \\
\hline Hostrock & Trachyte & & & & & & & & & & \\
\hline $\mathrm{SiO}_{2}($ wt. $\%$ ) & 66.31 & 66.09 & 61.35 & 64.31 & 63.40 & 61.06 & 63.27 & 63.03 & & & \\
\hline $\mathrm{Al}_{2} \mathrm{O}_{3}$ & 16.99 & 17.09 & 19.23 & 16.81 & 16.12 & 16.94 & 16.48 & 16.40 & & & \\
\hline $\mathrm{Fe}_{2} \mathrm{O}_{3}$ & 2.38 & 1.37 & 2.66 & 3.24 & 3.38 & 3.91 & 3.07 & 3.03 & & & \\
\hline $\mathrm{MgO}$ & 0.56 & 0.49 & 0.40 & 0.96 & 1.39 & 1.55 & 1.84 & 1.72 & & & \\
\hline $\mathrm{CaO}$ & 1.90 & 1.51 & 2.00 & 2.46 & 2.49 & 3.89 & 3.03 & 3.16 & & & \\
\hline $\mathrm{Na}_{2} \mathrm{O}$ & 4.68 & 5.24 & 5.10 & 5.09 & 4.81 & 5.34 & 5.12 & 5.17 & & & \\
\hline $\mathrm{K}_{2} \mathrm{O}$ & 5.33 & 5.04 & 6.03 & 5.43 & 6.34 & 5.25 & 5.80 & 5.80 & & & \\
\hline $\mathrm{TiO}_{2}$ & 0.53 & 0.41 & 0.40 & 0.48 & 0.50 & 0.50 & 0.38 & 0.37 & & & \\
\hline $\mathrm{P}_{2} \mathrm{O}_{5}$ & 0.29 & 0.19 & 0.22 & 0.26 & 0.30 & 0.28 & 0.18 & 0.23 & & & \\
\hline $\mathrm{MnO}$ & 0.02 & 0.01 & 0.03 & 0.06 & 0.06 & 0.09 & 0.18 & 0.06 & & & \\
\hline LOI & 0.8 & 2.2 & 2.1 & 0.7 & 0.8 & 0.6 & 0.1 & 0.2 & & & \\
\hline Total & 99.79 & 99.64 & 99.52 & 99.81 & 99.60 & 99.41 & 99.33 & 99.17 & & & \\
\hline Mg\# & 32.0 & 41.7 & 23.0 & 37.0 & 45.0 & 44.0 & 54.6 & 53.2 & & & \\
\hline $\mathrm{Sr}(\mathrm{ppm})$ & 1314.5 & 2241.6 & 2746.4 & 1373.7 & 2352.2 & 3616.0 & 3302.4 & 3470.8 & & & \\
\hline $\mathrm{Ba}$ & 1589.6 & 1179.8 & 2469.5 & 1379.1 & 2118.8 & 2488.6 & 2153.9 & 2277.0 & & & \\
\hline $\mathrm{Rb}$ & 173.9 & 129.6 & 187.5 & 191.0 & 202.7 & 158.6 & 144.3 & 147.0 & & & \\
\hline $\mathrm{Cs}$ & 5.7 & 6.9 & 12.3 & 5.9 & 6.9 & 2.6 & 2.3 & 1.7 & & & \\
\hline $\mathrm{Ga}$ & 21.4 & 21.4 & 25.4 & 21.2 & 22.4 & 25.5 & 22.2 & 24.1 & & & \\
\hline Hf & 8.7 & 6.6 & 13.1 & 9.5 & 11.1 & 11.3 & 9.1 & 9.6 & & & \\
\hline $\mathrm{Ni}$ & 9 & 27 & 5 & 5 & 28 & 12 & 15.1 & 14.9 & & & \\
\hline $\mathrm{Sc}$ & 5 & 4 & 3 & 4 & 5 & 5 & 4 & 3 & & & \\
\hline $\mathrm{Cr}$ & 6.8 & 34 & 7 & 7 & 34 & 21 & 27.4 & 34.2 & & & \\
\hline $\mathrm{Zr}$ & 357.8 & 285.4 & 523.9 & 353.5 & 431.4 & 460.4 & 319.8 & 294.5 & & & \\
\hline $\mathrm{Y}$ & 20.7 & 9.6 & 34.2 & 23.2 & 22.3 & 24.0 & 17.0 & 17.2 & & & \\
\hline $\mathrm{Nb}$ & 42.8 & 43.8 & 79.4 & 44.9 & 47.1 & 47.3 & 39.5 & 37.8 & & & \\
\hline $\mathrm{Ta}$ & 2.6 & 3.0 & 4.2 & 2.7 & 2.6 & 2.6 & 2.1 & 2.1 & & & \\
\hline $\mathrm{Th}$ & 45.1 & 38.5 & 98.4 & 55.2 & 65.1 & 78.6 & 61.0 & 63.1 & & & \\
\hline $\mathrm{U}$ & 10.7 & 14.7 & 28.0 & 16.1 & 17.6 & 18.2 & 17.7 & 19.7 & & & \\
\hline $\mathrm{Pb}$ & 6.5 & 5.3 & 10.9 & 34.7 & 22.2 & 4.3 & 19.3 & 5.6 & & & \\
\hline $\mathrm{La}$ & 91.5 & 85.1 & 211.3 & 119.4 & 158.8 & 165.9 & 121.5 & 126.8 & & & \\
\hline $\mathrm{Ce}$ & 155.8 & 144.1 & 350.2 & 199.6 & 262.4 & 348.3 & 265.4 & 274.9 & & & \\
\hline $\operatorname{Pr}$ & 16.03 & 14.00 & 32.75 & 18.95 & 25.74 & 30.95 & 23.68 & 24.56 & & & \\
\hline $\mathrm{Nd}$ & 50.7 & 44.6 & 101.3 & 61.8 & 82.1 & 102.5 & 83.3 & 84.8 & & & \\
\hline $\mathrm{Sm}$ & 7.6 & 6.3 & 13.1 & 8.5 & 11.0 & 14.4 & 9.7 & 10.2 & & & \\
\hline $\mathrm{Eu}$ & 1.74 & 1.93 & 3.29 & 2.05 & 2.92 & 3.39 & 2.53 & 2.81 & & & \\
\hline Gd & 4.13 & 2.89 & 7.40 & 5.04 & 5.85 & 7.67 & 5.29 & 5.75 & & & \\
\hline $\mathrm{Tb}$ & 0.66 & 0.41 & 1.10 & 0.77 & 0.85 & 1.01 & 0.70 & 0.75 & & & \\
\hline Dy & 3.59 & 2.12 & 5.17 & 4.00 & 3.83 & 4.46 & 3.20 & 3.35 & & & \\
\hline Ho & 0.64 & 0.31 & 1.04 & 0.66 & 0.64 & 0.70 & 0.51 & 0.53 & & & \\
\hline $\mathrm{Er}$ & 1.86 & 0.90 & 2.81 & 2.19 & 1.92 & 2.00 & 1.32 & 1.38 & & & \\
\hline $\mathrm{Tm}$ & 0.30 & 0.17 & 0.47 & 0.31 & 0.29 & 0.32 & 0.22 & 0.25 & & & \\
\hline $\mathrm{Yb}$ & 1.80 & 0.90 & 2.82 & 2.04 & 1.94 & 2.07 & 1.55 & 1.65 & & & \\
\hline $\mathrm{Lu}$ & 0.26 & 0.13 & 0.40 & 0.33 & 0.30 & 0.34 & 0.25 & 0.22 & & & \\
\hline$(\mathrm{La} / \mathrm{Sm})_{\mathrm{N}}$ & 7.58 & 8.50 & 10.15 & 8.84 & 9.09 & 7.25 & 7.88 & 7.82 & & & \\
\hline$(\mathrm{Gd} / \mathrm{Yb})_{\mathrm{N}}$ & 1.86 & 2.60 & 2.13 & 2.00 & 2.44 & 3.00 & 2.77 & 2.82 & & & \\
\hline $\mathrm{Eu} / \mathrm{Eu}^{*}$ & 0.95 & 1.38 & 1.40 & 0.96 & 1.11 & 0.98 & 1.08 & 1.21 & & & \\
\hline $\mathrm{La}_{\mathrm{N}}$ & 249 & 232 & 576 & 325 & 433 & 452 & 331 & 346 & & & \\
\hline $\mathrm{Yb}_{\mathrm{N}}$ & 7.3 & 3.6 & 11.4 & 8.2 & 7.8 & 8.3 & 6.3 & 6.7 & & & \\
\hline$(\mathrm{La} / \mathrm{Yb})_{\mathrm{N}}$ & 34.4 & 63.9 & 50.6 & 39.6 & 55.3 & 54.2 & 53.0 & 51.9 & & & \\
\hline $\mathrm{Ba} / \mathrm{Nb}$ & 37.14 & 26.93 & 31.10 & 30.71 & 44.99 & 52.61 & 54.53 & 60.24 & & & \\
\hline $\mathrm{La} / \mathrm{Nb}$ & 2.14 & 1.94 & 2.66 & 2.66 & 3.37 & 3.51 & 3.08 & 3.35 & & & \\
\hline $\mathrm{Rb} / \mathrm{Nb}$ & 4.1 & 3.0 & 2.4 & 4.3 & 4.3 & 3.4 & 3.7 & 3.9 & & & \\
\hline $\mathrm{Th} / \mathrm{Ta}$ & 17.45 & 12.83 & 23.43 & 20.44 & 25.04 & 30.23 & 29.05 & 50.05 & & & \\
\hline $\mathrm{Th} / \mathrm{Nb}$ & 1.05 & 0.88 & 1.24 & 1.23 & 1.38 & 1.66 & 1.54 & 1.67 & & & \\
\hline $\mathrm{Rb} / \mathrm{Sr}$ & 0.132 & 0.058 & 0.068 & 0.139 & 0.086 & 0.044 & 0.043 & 0.042 & & & \\
\hline Sample & GP-13 & Gl-5 & Gl-31 & Gl-57 & Gl-61 & Gl-67 & Gl-22 & Gl-38 & & & \\
\hline Host rock & Trachyte & \multicolumn{5}{|c|}{ Trachyandesite } & \multicolumn{5}{|c|}{ Basaltic-trachyandesite } \\
\hline $\mathrm{SiO}_{2}($ wt. $\%)$ & 60.53 & 57.89 & 56.79 & 55.22 & 57.11 & 57.10 & 50.38 & 50.64 & & & \\
\hline $\mathrm{Al}_{2} \mathrm{O}_{3}$ & 16.67 & 18.98 & 17.05 & 15.60 & 17.95 & 16.75 & 17.33 & 19.33 & & & \\
\hline
\end{tabular}


Table 1 (continued)

\begin{tabular}{|c|c|c|c|c|c|c|c|c|}
\hline$\overline{\mathrm{Fe}_{2} \mathrm{O}_{3}}$ & 3.77 & 3.86 & 4.42 & 5.41 & 4.80 & 4.51 & 6.72 & 5.45 \\
\hline $\mathrm{MgO}$ & 1.57 & 1.01 & 1.47 & 4.29 & 2.70 & 2.57 & 3.08 & 1.90 \\
\hline $\mathrm{CaO}$ & 3.89 & 3.16 & 5.36 & 6.95 & 4.96 & 5.50 & 7.63 & 1.41 \\
\hline $\mathrm{Na}_{2} \mathrm{O}$ & 5.41 & 4.14 & 4.26 & 3.14 & 4.72 & 3.75 & 2.28 & 1.80 \\
\hline $\mathrm{K}_{2} \mathrm{O}$ & 5.48 & 6.37 & 5.76 & 5.67 & 4.87 & 5.71 & 5.50 & 5.37 \\
\hline $\mathrm{TiO}_{2}$ & 0.47 & 0.42 & 0.53 & 0.67 & 0.59 & 0.51 & 0.70 & 1.66 \\
\hline $\mathrm{P}_{2} \mathrm{O}_{5}$ & 0.28 & 0.23 & 0.29 & 0.65 & 0.64 & 0.31 & 0.67 & 1.09 \\
\hline $\mathrm{MnO}$ & 0.09 & 0.07 & 0.10 & 0.09 & 0.09 & 0.09 & 0.13 & 0.02 \\
\hline LOI & 0.9 & 3.3 & 3.4 & 1.4 & 1.0 & 2.5 & 4.5 & 10.7 \\
\hline Total & 99.06 & 99.44 & 99.42 & 99.11 & 99.44 & 99.31 & 98.92 & 99.38 \\
\hline Mg\# & 45.5 & 34.4 & 40.0 & 61.0 & 53.0 & 53.0 & 47.9 & 41.0 \\
\hline $\mathrm{Sr}(\mathrm{ppm})$ & 3814.2 & 3096.9 & 3049.5 & 4978.8 & 3380.2 & 4203.9 & 5410.1 & 2970.7 \\
\hline $\mathrm{Ba}$ & 2591.8 & 2350.8 & 2770.3 & 3456.0 & 2502.0 & 2634.9 & 3409.5 & 2887.0 \\
\hline $\mathrm{Rb}$ & 154.6 & 163.7 & 147.8 & 139.2 & 101.0 & 134.7 & 92.2 & 138.2 \\
\hline $\mathrm{Cs}$ & 2.8 & 38.7 & 9.9 & 7.3 & 1.0 & 3.1 & 1.4 & 106.0 \\
\hline $\mathrm{Ga}$ & 24.7 & 22.4 & 20.9 & 21.5 & 22.5 & 20.1 & 20.2 & 21.7 \\
\hline Hf & 12.5 & 11.0 & 9.6 & 9.7 & 8.6 & 10.0 & 8.1 & 9.8 \\
\hline $\mathrm{Ni}$ & 4 & 5 & 5 & 23 & 19 & 15 & 6 & 95 \\
\hline $\mathrm{Sc}$ & 5 & 3 & 5 & 11 & 7 & 9 & 10 & 15 \\
\hline $\mathrm{Cr}$ & 3.4 & 6.8 & 7 & 48 & 21 & 41 & 7 & 21 \\
\hline $\mathrm{Zr}$ & 398.0 & 477.9 & 388.6 & 414.6 & 393.6 & 395.7 & 376.6 & 419.0 \\
\hline $\mathrm{Y}$ & 23.2 & 23.6 & 26.4 & 25.0 & 22.9 & 24.3 & 28.6 & 33.9 \\
\hline $\mathrm{Nb}$ & 47.2 & 73.3 & 43.3 & 54.1 & 54.8 & 50.2 & 47.7 & 64.7 \\
\hline $\mathrm{Ta}$ & 2.5 & 4.4 & 2.5 & 3.0 & 3.4 & 3.1 & 3.2 & 3.8 \\
\hline $\mathrm{Th}$ & 77.2 & 71.4 & 57.6 & 68.4 & 45.1 & 57.5 & 59.0 & 35.6 \\
\hline $\mathrm{U}$ & 18.9 & 13.5 & 13.2 & 16.8 & 10.5 & 15.5 & 11.4 & 4.9 \\
\hline $\mathrm{Pb}$ & 3.6 & 10.6 & 9.3 & 5.8 & 9.6 & 13.3 & 6.4 & 6.5 \\
\hline $\mathrm{La}$ & 152.0 & 211.6 & 197.1 & 228.0 & 185.5 & 191.3 & 273.4 & 112.6 \\
\hline $\mathrm{Ce}$ & 350.4 & 374.6 & 345.4 & 430.5 & 317.7 & 324.5 & 495.9 & 214.0 \\
\hline $\mathrm{Pr}$ & 30.69 & 36.45 & 33.86 & 44.92 & 31.31 & 31.49 & 50.54 & 23.83 \\
\hline $\mathrm{Nd}$ & 109.3 & 111.8 & 111.9 & 154.4 & 99.5 & 98.4 & 174.6 & 91.9 \\
\hline $\mathrm{Sm}$ & 13.2 & 13.3 & 13.9 & 19.5 & 13.2 & 13.1 & 22.7 & 16.2 \\
\hline $\mathrm{Eu}$ & 3.14 & 3.15 & 3.47 & 5.04 & 3.43 & 3.41 & 5.70 & 4.53 \\
\hline $\mathrm{Gd}$ & 7.52 & 5.89 & 6.66 & 9.00 & 6.65 & 6.56 & 10.54 & 10.57 \\
\hline $\mathrm{Tb}$ & 0.94 & 0.93 & 1.02 & 1.24 & 0.94 & 0.94 & 1.30 & 1.44 \\
\hline Dy & 4.20 & 4.02 & 4.65 & 5.04 & 4.61 & 4.71 & 5.38 & 6.44 \\
\hline Но & 0.67 & 0.73 & 0.80 & 0.78 & 0.74 & 0.72 & 0.82 & 1.15 \\
\hline $\mathrm{Er}$ & 2.03 & 1.95 & 2.34 & 1.98 & 1.95 & 2.18 & 2.11 & 2.96 \\
\hline $\mathrm{Tm}$ & 0.31 & 0.34 & 0.38 & 0.31 & 0.30 & 0.32 & 0.37 & 0.40 \\
\hline $\mathrm{Yb}$ & 2.08 & 1.84 & 2.29 & 2.03 & 1.85 & 2.33 & 2.18 & 2.31 \\
\hline $\mathrm{Lu}$ & 0.32 & 0.30 & 0.33 & 0.27 & 0.31 & 0.32 & 0.31 & 0.36 \\
\hline$(\mathrm{La} / \mathrm{Sm})_{\mathrm{N}}$ & 7.25 & 10.01 & 8.93 & 7.36 & 8.85 & 9.9 & 7.58 & 4.37 \\
\hline$(\mathrm{Gd} / \mathrm{Yb})_{\mathrm{N}}$ & 2.93 & 2.59 & 2.36 & 3.60 & 2.91 & 2.28 & 3.92 & 3.71 \\
\hline $\mathrm{Eu} / \mathrm{Eu}^{*}$ & 0.96 & 1.10 & 1.10 & 1.16 & 1.12 & 1.12 & 1.12 & 1.05 \\
\hline $\mathrm{La}_{\mathrm{N}}$ & 414 & 577 & 537 & 621 & 505 & 521 & 745 & 307 \\
\hline $\mathrm{Yb}_{\mathrm{N}}$ & 8.4 & 7.4 & 9.2 & 8.2 & 7.5 & 9.4 & 8.8 & 9.3 \\
\hline$(\mathrm{La} / \mathrm{Yb})_{\mathrm{N}}$ & 49.4 & 77.7 & 58.1 & 75.9 & 67.8 & 55.5 & 84.7 & 32.9 \\
\hline $\mathrm{Ba} / \mathrm{Nb}$ & 54.91 & 32.07 & 63.98 & 63.88 & 45.66 & 52.49 & 71.48 & 44.62 \\
\hline $\mathrm{La} / \mathrm{Nb}$ & 3.22 & 2.89 & 4.55 & 4.21 & 3.39 & 3.81 & 5.73 & 1.74 \\
\hline $\mathrm{Rb} / \mathrm{Nb}$ & 3.3 & 2.2 & 3.4 & 2.6 & 1.8 & 2.7 & 1.9 & 2.1 \\
\hline $\mathrm{Th} / \mathrm{Ta}$ & 30.88 & 16.23 & 23.04 & 22.80 & 13.26 & 18.55 & 18.44 & 9.37 \\
\hline $\mathrm{Th} / \mathrm{Nb}$ & 1.65 & 0.97 & 1.33 & 1.26 & 0.82 & 1.15 & 1.24 & 0.55 \\
\hline $\mathrm{Rb} / \mathrm{Sr}$ & 0.040 & 0.053 & 0.048 & 0.028 & 0.030 & 0.032 & 0.017 & 0.047 \\
\hline Sample & Gl-40 & Gl-48 & Gl-55 & Gl-70 & Gl-81 & Gl-96 & Gl-109 & \\
\hline Hostrock & \multicolumn{8}{|c|}{ Basaltic-trachyandesite } \\
\hline $\mathrm{SiO}_{2}($ wt. $\%)$ & 50.18 & 51.29 & 51.92 & 47.81 & 50.60 & 51.19 & 51.12 & \\
\hline $\mathrm{Al}_{2} \mathrm{O}_{3}$ & 16.34 & 16.09 & 16.51 & 17.14 & 16.15 & 15.98 & 17.43 & \\
\hline $\mathrm{Fe}_{2} \mathrm{O}_{3}$ & 6.17 & 5.59 & 6.01 & 7.14 & 5.79 & 6.50 & 6.27 & \\
\hline $\mathrm{MgO}$ & 4.33 & 3.57 & 4.52 & 4.85 & 3.44 & 2.87 & 3.59 & \\
\hline $\mathrm{CaO}$ & 6.46 & 9.12 & 8.25 & 7.76 & 9.06 & 7.51 & 8.18 & \\
\hline $\mathrm{Na}_{2} \mathrm{O}$ & 1.84 & 2.22 & 2.74 & 2.25 & 3.24 & 1.63 & 4.56 & \\
\hline $\mathrm{K}_{2} \mathrm{O}$ & 5.06 & 5.74 & 4.82 & 3.64 & 4.56 & 4.44 & 3.30 & \\
\hline $\mathrm{TiO}_{2}$ & 1.41 & 0.82 & 0.78 & 1.47 & 0.65 & 0.97 & 0.71 & \\
\hline $\mathrm{P}_{2} \mathrm{O}_{5}$ & 1.05 & 0.69 & 0.64 & 1.11 & 0.61 & 0.87 & 0.70 & \\
\hline
\end{tabular}


Table 1 (continued)

\begin{tabular}{|c|c|c|c|c|c|c|c|}
\hline $\mathrm{MnO}$ & 0.07 & 0.11 & 0.11 & 0.09 & 0.12 & 0.11 & 0.12 \\
\hline LOI & 6.5 & 4.0 & 2.8 & 6.0 & 5.1 & 6.5 & 3.0 \\
\hline Total & 99.42 & 99.25 & 99.11 & 99.28 & 99.32 & 98.57 & 98.98 \\
\hline $\mathrm{Mg \#}$ & 58.0 & 56.0 & 60.0 & 57.7 & 54.0 & 46.9 & 53.0 \\
\hline $\mathrm{Sr}(\mathrm{ppm})$ & 3143.7 & 4362.8 & 5390.0 & 3067.6 & 3530.8 & 6535.9 & 5678.5 \\
\hline $\mathrm{Ba}$ & 2418.2 & 2951.0 & 2835.0 & 2910.9 & 3495.6 & 5351.6 & 3586.2 \\
\hline $\mathrm{Rb}$ & 118.5 & 149.6 & 102.7 & 42.4 & 107.2 & 28.8 & 130.9 \\
\hline Cs & 1.5 & 3.8 & 1.7 & 0.7 & 3.9 & 7.5 & 3.4 \\
\hline $\mathrm{Ga}$ & 18.9 & 19.3 & 19.1 & 21.2 & 21.2 & 23.1 & 22.2 \\
\hline Hf & 8.3 & 10.1 & 7.8 & 9.9 & 9.3 & 20.4 & 9.3 \\
\hline $\mathrm{Ni}$ & 54 & 12 & 19 & 58 & 10 & 8 & 13 \\
\hline $\mathrm{Sc}$ & 13 & 11 & 14 & 15 & 13 & 9 & 12 \\
\hline $\mathrm{Cr}$ & 34 & 14 & 68 & 34 & 34 & 14 & 14 \\
\hline $\mathrm{Zr}$ & 360.2 & 427.5 & 336.9 & 406.6 & 386.1 & 817.3 & 415.6 \\
\hline $\mathrm{Y}$ & 25.7 & 30.4 & 25.7 & 26.2 & 28.3 & 40.5 & 29.3 \\
\hline $\mathrm{Nb}$ & 55.8 & 47.4 & 45.3 & 61.8 & 50.7 & 90.9 & 51.8 \\
\hline $\mathrm{Ta}$ & 3.1 & 2.7 & 2.9 & 3.5 & 3.1 & 5.0 & 3.4 \\
\hline Th & 31.2 & 48.4 & 43.6 & 33.8 & 55.2 & 66.4 & 66.6 \\
\hline $\mathrm{U}$ & 4.7 & 12.7 & 8.8 & 5.3 & 13.1 & 4.7 & 14.0 \\
\hline $\mathrm{Pb}$ & 6.0 & 6.1 & 3.7 & 8.1 & 11.3 & 18.7 & 1.7 \\
\hline $\mathrm{La}$ & 165.3 & 223.6 & 196.1 & 174.9 & 189.3 & 317.4 & 297.6 \\
\hline $\mathrm{Ce}$ & 312.9 & 405.5 & 354.3 & 325.5 & 345.2 & 610.5 & 550.7 \\
\hline $\operatorname{Pr}$ & 32.46 & 42.29 & 36.17 & 33.62 & 33.89 & 62.36 & 54.21 \\
\hline $\mathrm{Nd}$ & 114.3 & 145.3 & 118.5 & 113.7 & 115.0 & 215.4 & 188.7 \\
\hline $\mathrm{Sm}$ & 16.2 & 19.3 & 16.4 & 16.5 & 16.4 & 30.4 & 23.5 \\
\hline $\mathrm{Eu}$ & 4.17 & 4.83 & 4.15 & 4.48 & 3.98 & 7.69 & 5.79 \\
\hline $\mathrm{Gd}$ & 8.78 & 9.55 & 8.41 & 9.35 & 8.50 & 15.13 & 10.08 \\
\hline $\mathrm{Tb}$ & 1.18 & 1.25 & 1.22 & 1.13 & 1.16 & 2.11 & 1.25 \\
\hline Dy & 5.29 & 5.81 & 5.25 & 5.50 & 5.38 & 8.07 & 5.61 \\
\hline Ho & 0.90 & 0.93 & 0.84 & 0.88 & 0.87 & 1.18 & 0.93 \\
\hline Er & 1.99 & 2.50 & 2.15 & 2.21 & 2.36 & 3.04 & 2.26 \\
\hline $\mathrm{Tm}$ & 0.31 & 0.36 & 0.35 & 0.31 & 0.32 & 0.44 & 0.36 \\
\hline $\mathrm{Yb}$ & 1.65 & 2.24 & 2.09 & 2.11 & 2.17 & 2.80 & 2.36 \\
\hline $\mathrm{Lu}$ & 0.25 & 0.33 & 0.31 & 0.26 & 0.34 & 0.44 & 0.34 \\
\hline$(\mathrm{La} / \mathrm{Sm})_{\mathrm{N}}$ & 6.42 & 7.29 & 7.53 & 6.67 & 7.27 & 6.57 & 7.97 \\
\hline$(\mathrm{Gd} / \mathrm{Yb})_{\mathrm{N}}$ & 4.31 & 3.46 & 3.26 & 3.59 & 3.17 & 4.38 & 3.46 \\
\hline $\mathrm{Eu} / \mathrm{Eu}^{*}$ & 1.07 & 1.09 & 1.08 & 1.10 & 1.03 & 1.09 & 1.15 \\
\hline $\mathrm{La}_{\mathrm{N}}$ & 450 & 609 & 534 & 477 & 516 & 865 & 811 \\
\hline $\mathrm{Yb}_{\mathrm{N}}$ & 6.7 & 9.0 & 8.4 & 8.5 & 8.8 & 11.3 & 9.5 \\
\hline$(\mathrm{La} / \mathrm{Yb})_{\mathrm{N}}$ & 67.7 & 67.5 & 63.4 & 56.0 & 58.9 & 76.6 & 85.2 \\
\hline $\mathrm{Ba} / \mathrm{Nb}$ & 43.34 & 62.26 & 62.58 & 47.10 & 68.95 & 58.87 & 69.23 \\
\hline $\mathrm{La} / \mathrm{Nb}$ & 2.96 & 4.72 & 4.33 & 2.83 & 3.73 & 3.49 & 5.75 \\
\hline $\mathrm{Rb} / \mathrm{Nb}$ & 2.1 & 3.2 & 2.3 & 0.7 & 2.1 & 0.3 & 2.5 \\
\hline $\mathrm{Th} / \mathrm{Ta}$ & 10.06 & 17.93 & 15.03 & 9.66 & 17.81 & 13.28 & 19.59 \\
\hline $\mathrm{Th} / \mathrm{Nb}$ & 0.56 & 1.02 & 0.96 & 0.55 & 1.09 & 0.73 & 1.29 \\
\hline $\mathrm{Rb} / \mathrm{Sr}$ & 0.038 & 0.034 & 0.019 & 0.014 & 0.030 & 0.004 & 0.023 \\
\hline
\end{tabular}

